\title{
Doppler echocardiography in the study of patients with mitral disc valve prostheses
}

\author{
SIGURD NITTER-HAUGE \\ From the Medical Department B, Laboratory of Cardiology, University Hospital, Rikshospitalet, Oslo, Norway
}

SUMMARY A combination of M mode and Doppler echocardiography was used to study patients with mitral disc valve prostheses. The probe used in these investigations consisted of a circular Doppler crystal mounted around the $M$ mode crystal in the same plane. Because of the strong echoes produced by the prosthesis the transducer (probe) could be angled for optimum Doppler signals without losing the $M$ mode echocardiographic recording of the prosthesis. With this equipment mean and maximum blood velocities and Doppler amplitude signals could be measured simultaneously with $\mathbf{M}$ mode echocardiography. A depth indication line in the $\mathbf{M}$ mode recording ensured that the Doppler signal was recorded in the region of interest. The Doppler ultrasound technique was also used separately in both the pulsed wave and the continuous wave mode.

The data show the usefulness of this technique in patients with normally functioning valve prostheses and in three patients with valve malfunction due to thrombus formation. The data in the latter three cases seem to indicate that the Doppler technique provides valuable information in addition to that obtained by $\mathbf{M}$ mode echocardiography in recognising mitral valve prosthetic malfunction.

Since the introduction of prosthetic heart valves the search has continued for non-invasive methods ${ }^{12}$ that can provide a useful technique for routine postoperative assessment. A necessary prerequisite for such methods is that they are able to identify both normally functioning prostheses as well as those with varying degrees of malfunction. In this respect, valve thrombus formation is of particular interest since at present it represents the major threat to the patient's life. This report reviews experience with a technique using Doppler ultrasound combined with $M$ mode echocardiography in assessing patients with different types of mitral disc valve prostheses. This technique has been used in this department for the past two years and appears to be of considerable diagnostic value.

\section{Patients and methods}

The ultrasonic Doppler instrument used ${ }^{3-5}$ is a slightly modified version of the commercially available Doppler ultrasound system (PEDOF, Vingmed $\mathrm{A} / \mathrm{S})$. The ultrasonic frequency is $2 \mathrm{MHz}$. The instrument has a non-directional maximum frequency

Accepted for publication 23 August 1983 estimator and a mean frequency estimator that also gives the direction of flow (positive, flow towards the transducer; negative, away from the transducer). The amplitude of the Doppler signal is recorded separately. The valve movements give strong reflections and are heard as loud clicks, whereas blood flow produces a more continuous and less intense sound. The valve movements, therefore, show up as spikes in the amplitude of the Doppler signal. The amplitude of the Doppler signal is, therefore, useful for timing the valve movements as well as for localising the sample volume.

For a signal to pass through the instrument's high pass filter a certain velocity is required. A cutoff frequency of $500 \mathrm{~Hz}$ is most often used. When low velocities are present a low frequency filter is used, whereas with high velocities a more high frequency filter can be used. For the best results the level of the signal before it enters the estimator should be adjusted, so that the estimator is barely tracking the maximum signal frequency, to avoid the possibility of the estimator locking on to the noise. With the PEDOF system a lamp lights up when a sufficient signal level at the estimator input is reached. A low value of the gain is used initially and is then increased so that the lamp lights up. To ensure that the 


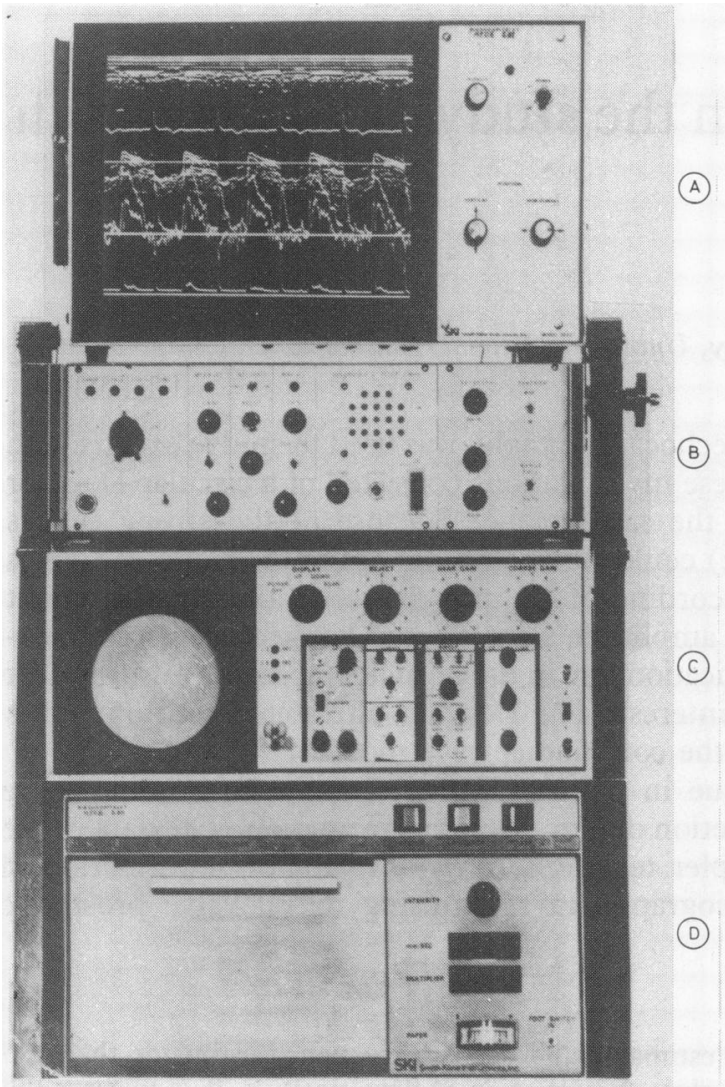

Fig. 1 Combined pulsed Doppler ultrasound recorder $(B)$ (modified model of PEDOF-Vingmed $A / S$ ) and $M$ mode echocardiograph $(C)$ (Smith Kline ultrasonoscope) with monitor (A) and fibre optic recorder (D).

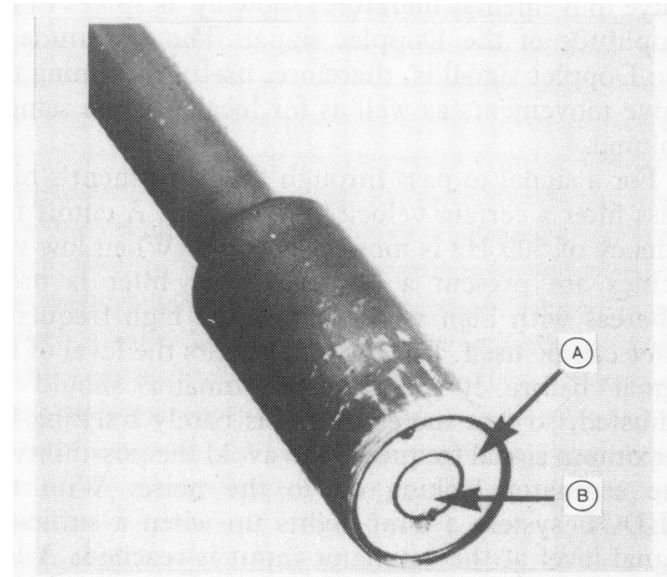

Fig. 2 Transducer (probe) with crystal for pulsed Doppler ultrasound $(A)$ and crystal for pulsed echocardiography $(B)$. estimator is producing correct results it is necessary to check that the estimator output is independent of some increasing input gain adjustment after the lamp has lit and before the output takes off the noise.

The instrument may be used either in a pulsed mode or as a continuous wave velocity meter. Using repetition frequencies of 9.8 and $6.7 \mathrm{kHz}$, velocities up to $1.7 \mathrm{~m} / \mathrm{s}$ may be measured within $7 \mathrm{~cm}$ and up to $1 \mathrm{~m} / \mathrm{s}$ within $12 \mathrm{~cm}$ of the transducer. When used in the continuous wave mode, velocities up to $7 \mathrm{~m} / \mathrm{s}$ may be measured but with a loss of range resolution.

For this study of mitral valve prostheses, the Doppler ultrasound equipment was combined with an $M$ mode echocardiograph (Smith Kline) ultrasonoscope (Fig. 1). The transducer included a circular Doppler crystal mounted around an $M$ mode echo crystal in the same plane (Fig. 2). The Doppler was provided with a depth indication line in the $M$ mode echo recordings which ensured that the Doppler signal was recorded in the optimal part of the region of interest. For simultaneous measurements the Doppler system could be used in the pulsed mode only. When used in the continuous mode the $\mathbf{M}$ mode echo was automatically switched off, and high velocity jets could be obtained with the Doppler.

The transducer was placed in the third to fourth intercostal space slightly medial to the apex of the heart and angled until the characteristic sound of the mitral flow was detected. The direction of the transducer was carefully adjusted until the maximum Doppler shift was found. As the prosthetic valve produced strong echoes the transducer could be angled for optimum Doppler signals without losing the $M$ mode echocardiographic record of the prosthesis, although the valve housing could be reduced in intensity. The maximum and mean velocities and the $M$ mode echocardiogram were recorded either simultaneously or separately as described above. The PEDOF is calibrated with an audiotone of $2.0 \mathrm{kHz}$, which is equivalent to a blood velocity of $1 \mathrm{~m} / \mathrm{s}$ if the ultrasound beam is in line with the flow and equivalent to a pressure gradient of $4 \mathrm{~mm} \mathrm{Hg}$ from the simplified Bernoulli equation. 6

\section{Results}

\section{NORMALLY FUNCTIONING PROSTHESIS}

Case 1

A 64 year old woman with rheumatic heart disease was seen one year after implantation of a mitral valve prosthesis (Björk-Shiley No 29). She was in NYHA class $I$, in sinus rhythm with no systolic murmur and with distinct prosthetic valve clicks; there were no radiographic signs of prosthetic malfunction; Fig 3 shows ultrasound recordings. 


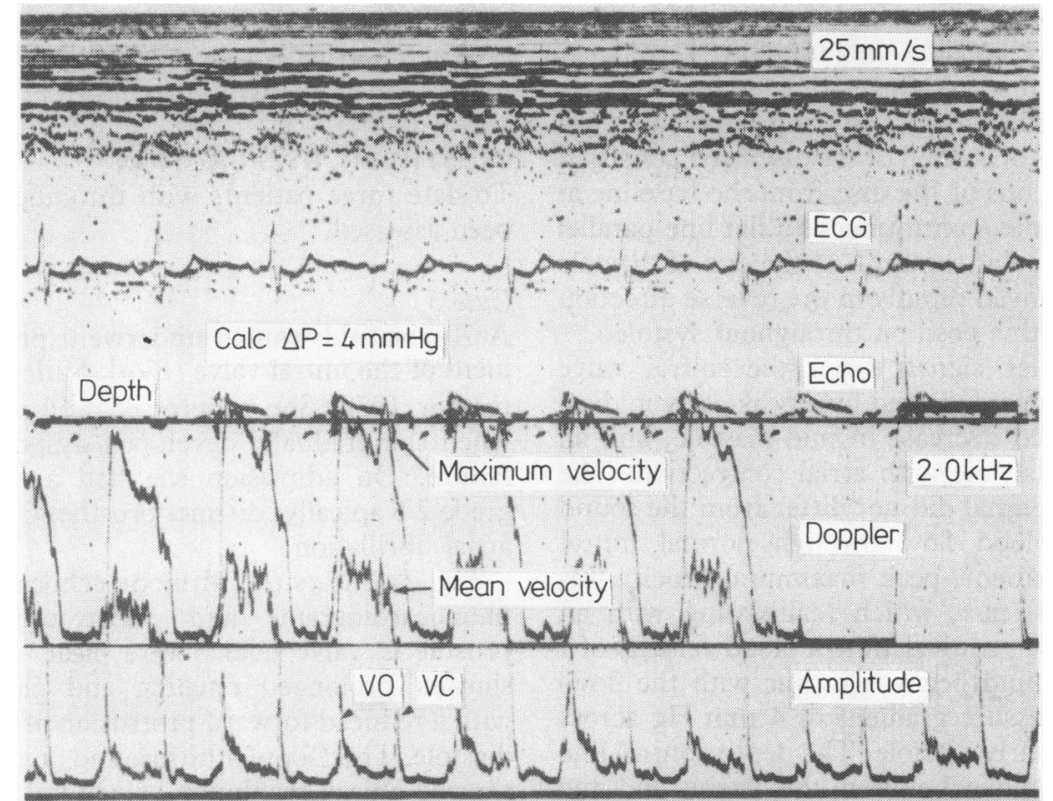

Fig. 3 Simultaneous pulsed $M$ mode echocardiogram and pulsed Doppler recordings in a patient (case 1) with a normally functioning Björk-Shiley mitral disc valve prosthesis (No 29) showing sinus rhythm. Calculated $\Delta P$ denotes the pressure gradient across the mitral valve prosthesis when calibrated with an audiotone of $2.0 \mathrm{kHz}$ which is equivalent to a blood velocity of $1 \mathrm{~ms}$ if the ultrasound beam is in line with the flow and equivalent to a pressure gradient of $4 \mathrm{~mm} \mathrm{Hg}$ from the simplified Bernoulli equation. ${ }^{6}$ $V O$, mitral valve opening; VC, mitral valve closure; ECG, electrocardiogram.

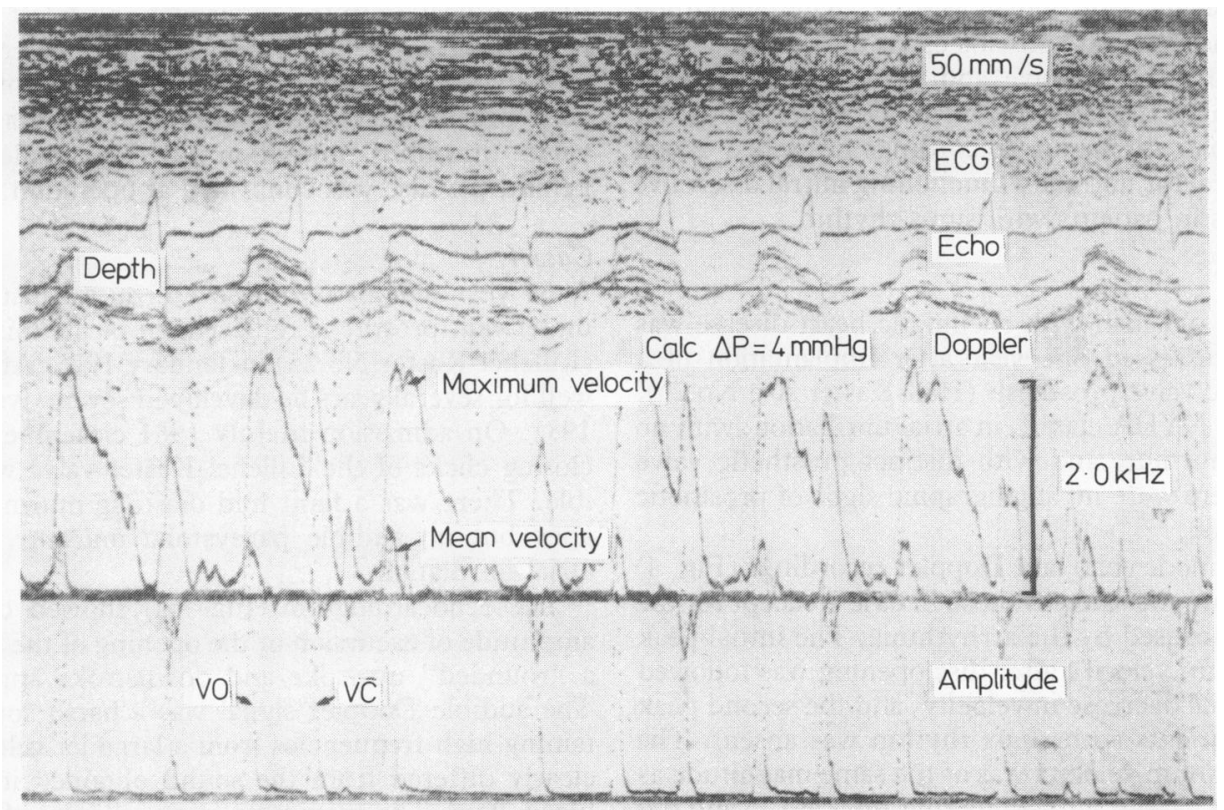

Fig. 4 Simultaneous pulsed $M$ mode echocardiogram and pulsed Doppler recordings in a patient (case 2) with normally functioning Hall-Kaster disc valve prosthesis (No 29) showing atrial fibrillation. VO, mitral valve opening; VC, mitral valve closure; ECG, electrocardiogram; Calc $\Delta P$, calculated $\Delta P$ (as in Fig. 3). 
The echocardiogram showed heavy echoes produced by the valve housing, including the valve ring, moving towards the transducer during atrial systole and away from the transducer during ventricular systole. The patterns of motion of the disc itself consisted of abrupt separations of the disc from the baseline at the onset of diastole, continuing as a flat line parallel to the interventricular septum. At the end of diastole the disc echoes moved rapidly in the reverse direction and remained in this position throughout systole.

Normal Doppler signals from the mitral valve outflow tract are characterised by a peak early in diastole, a pronounced decrease in mid-diastole, and an increase in late diastole with atrial contraction. The audible Doppler signal did not differ from the sound obtained from blood flow through normal mitral ostium. In this patient, peak maximum velocity was measured to $1.00 \mathrm{~m} / \mathrm{s}$, which (calibrating with an audiotone of $2 \mathrm{kHz}$, equivalent to a blood velocity of 1 $\mathrm{m} / \mathrm{s}$ if the ultrasound beam is in line with the flow) means a peak pressure gradient of $4 \mathrm{~mm} \mathrm{Hg}$ across the prosthesis in early diastole. The depth control line indicates the distance between the probe and the structures from which the ultrasound is reflected and ensures that the Doppler signal is recorded from the mitral area. The mean velocity, which is directional, is towards the tranducer (positive) in diastole. There is no flow from the transducer during systole, indicating no regurgitation from the left ventricle to the left atrium during ventricular systole. The amplitude records the opening and closing of the valve.

Based on non-invasive and invasive studies of a large number of cases, the $M$ mode echocardiogram and Doppler ultrasound recordings described above were typical for normally functioning mitral disc valve prostheses in patients with sinus rhythm.

\section{Case 2}

A 59 year old man with rheumatic heart disease was routinely assessed one year after implantation of a mitral disc valve prosthesis (Hall-Kaster size No 29). He was in NYHA class $I$, in atrial fibrillation, with no systolic murmur, and with distinct prosthetic valve clicks; there were no angiographic signs of prosthetic malfunction.

The $M$ mode echo and Doppler recordings (Fig. 4) were mainly the same as those in case 1 except for the variations caused by the arrhythmia. The initial peak in maximum velocity after disc opening was followed by a gradual decrease in velocity, and the second peak seen in patients with sinus rhythm was absent. The peak maximum velocity was of the same magnitude as that in the patient with sinus rhythm (case 1) and did not vary with the duration of diastole. If diastole is long with ventricular rate, the initial high maximum velocity is gradually reduced to almost zero indicating a small flow at the end of diastole. This curve was typical of normally functioning mitral disc valve prostheses in patients with atrial fibrillation.

\section{PROSTHETIC DYSFUNCTION}

To date three patients with thrombosed valves have been assessed.

\section{Case 3}

A 70 year old woman underwent prosthetic replacement of the mitral valve (Björk-Shiley No 29) in September 1978. She progressed well until June 1981, when she gradually developed dyspnoea and cardiac failure. On admission she had a systolic murmur grade $2 / 5$ apically, distinct prosthetic valve clicks, and atrial fibrillation.

Fig. 5a shows the $M$ mode echocardiogram with a phonocardiogram and electrocardiogram. The prosthetic valve clicks were clear. The valve echo showed prolonged opening and closing velocities, with a reduced forward protrusion of the valve during diastole. The Doppler ultrasound (Fig. 5b) recordings showed a peak maximum velocity of $2.25 \mathrm{~m} / \mathrm{s}$ corresponding to a fall in peak pressure of $20 \mathrm{~mm} \mathrm{Hg}$. Thus peak maximum velocity and fall in peak pressure were greater than those in patients with normally functioning mitral disc valve prostheses. The mean velocity flow was towards the transducer in diastole (positive) but away from the transducer during systole (negative), indicating mitral regurgitation. A left ventricular angiogram recorded a few days later showed impaired opening and closing movements of the disc with prosthetic valve regurgitation. A diagnosis of valve thrombus formation at the smaller orifice behind the disc was confirmed at operation.

\section{Case 4}

A 64 year old man with severe mitral insufficiency underwent prosthetic replacement of the mitral valve (Lillehei-Kaster No 25) in January 1975. After being well for several years he developed severe dyspnoea in 1981. On admission in July 1981 clear opening and closing clicks of the Lillehei-Kaster valve were audible. There was a faint mid-diastolic murmur at the apex but no audible pansystolic murmur. He had atrial fibrillation.

The echocardiogram (Fig. 6a) showed decreased amplitude of excursion of the opening of the disc with a "rounded" upstroke and downstroke appearance. The audible Doppler signal was a harsh sound containing high frequencies from a large jet velocity and clearly differed from the sound obtained in normal blood flow. The maximum peak velocity after valve opening exceeded $1.0 \mathrm{~m} / \mathrm{s}$ for the pulsed mode of the instrument. The Doppler recordings were therefore made in continuous wave mode (Fig. 6b). The peak 
Doppler echocardiography in the study of patients with mitral disc valve prostheses
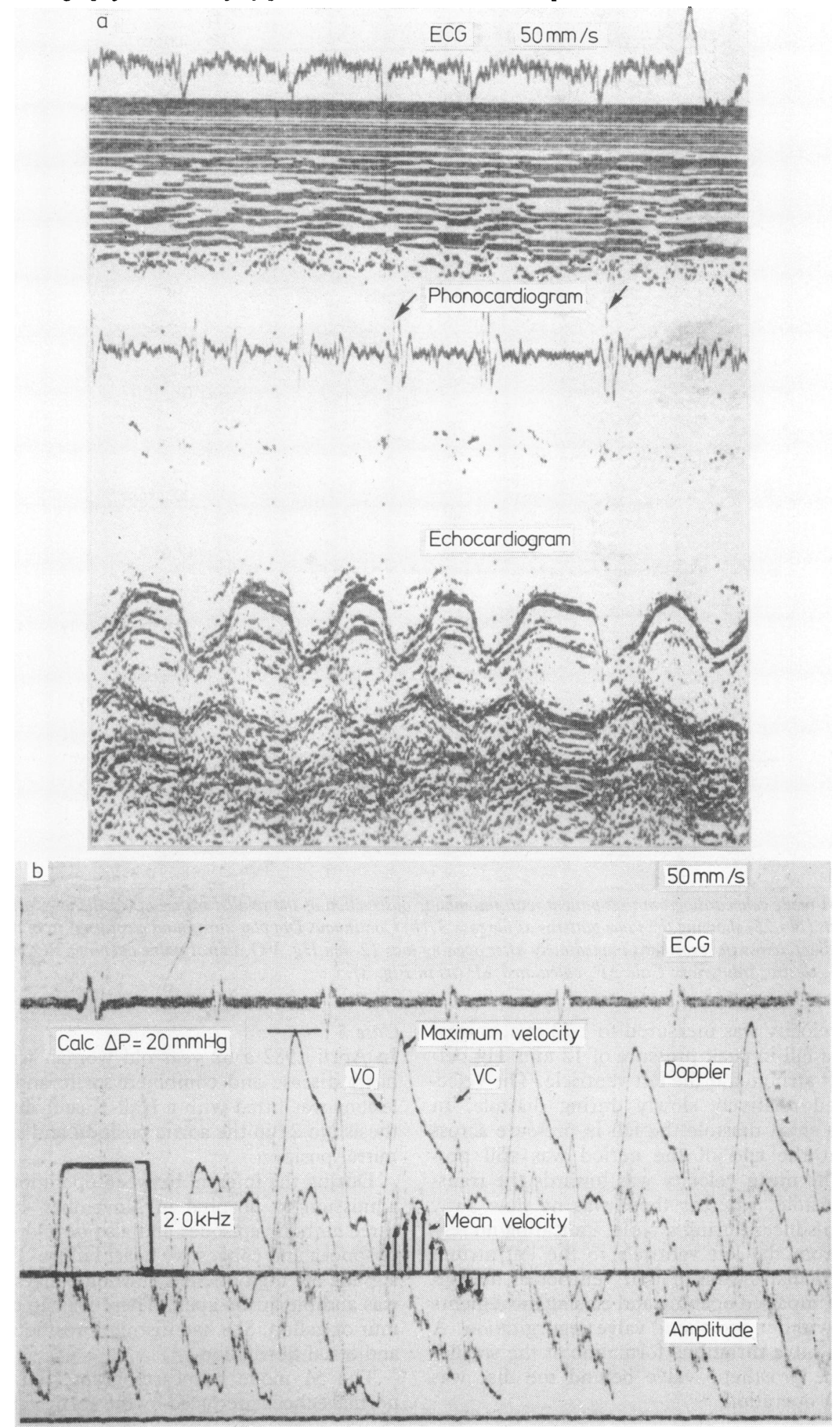

Fig. 5 (a) Simultaneous $M$ mode echocardiogram and phonocardiogram in a patient (case 3) with thrombotic obstruction of the smaller orifice of a Björk-Shiley mitral disc valve prosthesis (No 29). Valve opening and closing is delayed; the delay in valve movements is associated with abnormal forward motion of the prosthetic cage. Note the closing sound of the disc on the phonocardiogram (arrow). (b) Continuous Doppler ultrasound recordings from the same patient. The peak gradient across the prosthesis immediately after opening was $20 \mathrm{~mm} \mathrm{Hg}$. VO, mitral valve opening; VC, mitral valve closure; ECG, electrocardiogram; Calc $\Delta P$, calculated $\Delta P$ (as in Fig. 3). 


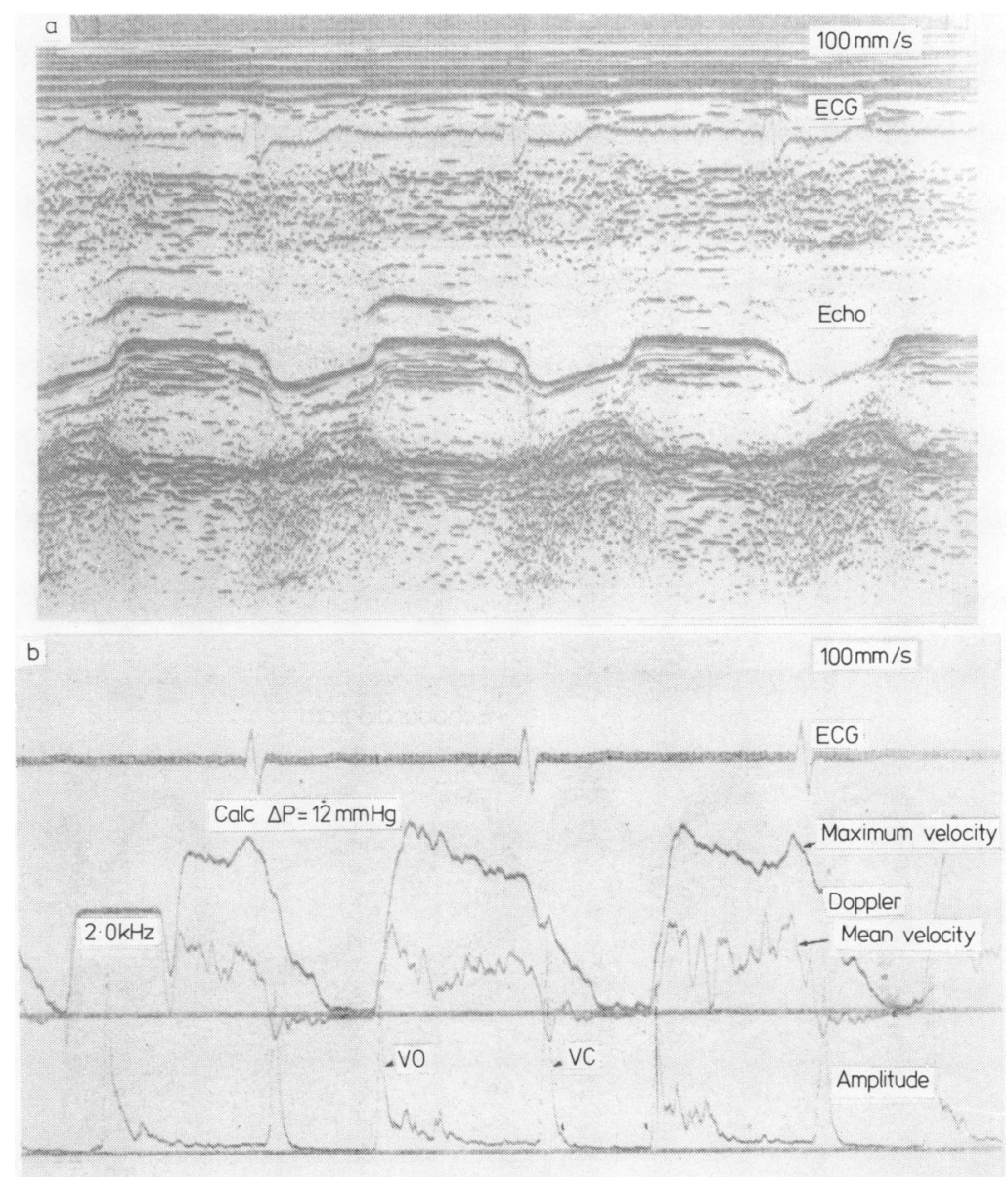

Fig. 6 (a) M mode echocardiogram in a patient with thrombotic obstruction of the smaller orifice of a Lillehei-Kaster mitral disc valve prosthesis (No 25) showing the same patterns as for case 3. (b) Continuous Doppler ultrasound recordings from the same patient. The peak gradient across the prosthesis immediately after opening was $12 \mathrm{~mm} \mathrm{Hg}$. VO, mitral valve opening; VC, mitral valve closure; ECG, electrocardiogram; Calc $\Delta P$, calculated $\Delta P$ (as in Fig. 3).

maximum velocity was measured to $1.72 \mathrm{~m} / \mathrm{s}$, corresponding to a fall in peak pressure of $12 \mathrm{~mm} \mathrm{Hg}$ between the left atrium and the left ventricle. The velocity decreased relatively slowly during diastole. In beats with a short diastole the fall in pressure across the valve at the end of the period was still pronounced. The mean velocity was towards the transducer in diastole, whereas there was no flow away from the transducer during systole, indicating no regurgitation from the left ventricle to the left atrium during ventricular systole. A left ventricular angiogram showed impaired opening and closing movements of the disc without prosthetic valve regurgitation. A diagnosis of valve thrombus formation at the smaller orifice of the prosthetic valve behind the disc was confirmed at operation.

\section{Case 5}

In April 1982 a 62 year old woman with rheumatic heart disease and combined aortic and mitral valve lesions was fitted with a Hall-Kaster disc valve prosthesis No 21 in the aortic position and a No 29 in the mitral position.

During the interval between operation and the last admission to hospital in November 1981, she had three embolic episodes and also developed increasing dyspnoea and congestive heart failure. On admission, a grade $2 / 6$ high pitched blowing pansystolic murmur was audible at the apex. There was no diastolic murmur or gallop. She had distinct prosthetic valve clicks and atrial fibrillation.

The $M$ mode echocardiogram (Fig. 7a) showed normal echoes produced by the aortic valve and mitral 
Doppler echocardiography in the study of patients with mitral disc valve prostheses
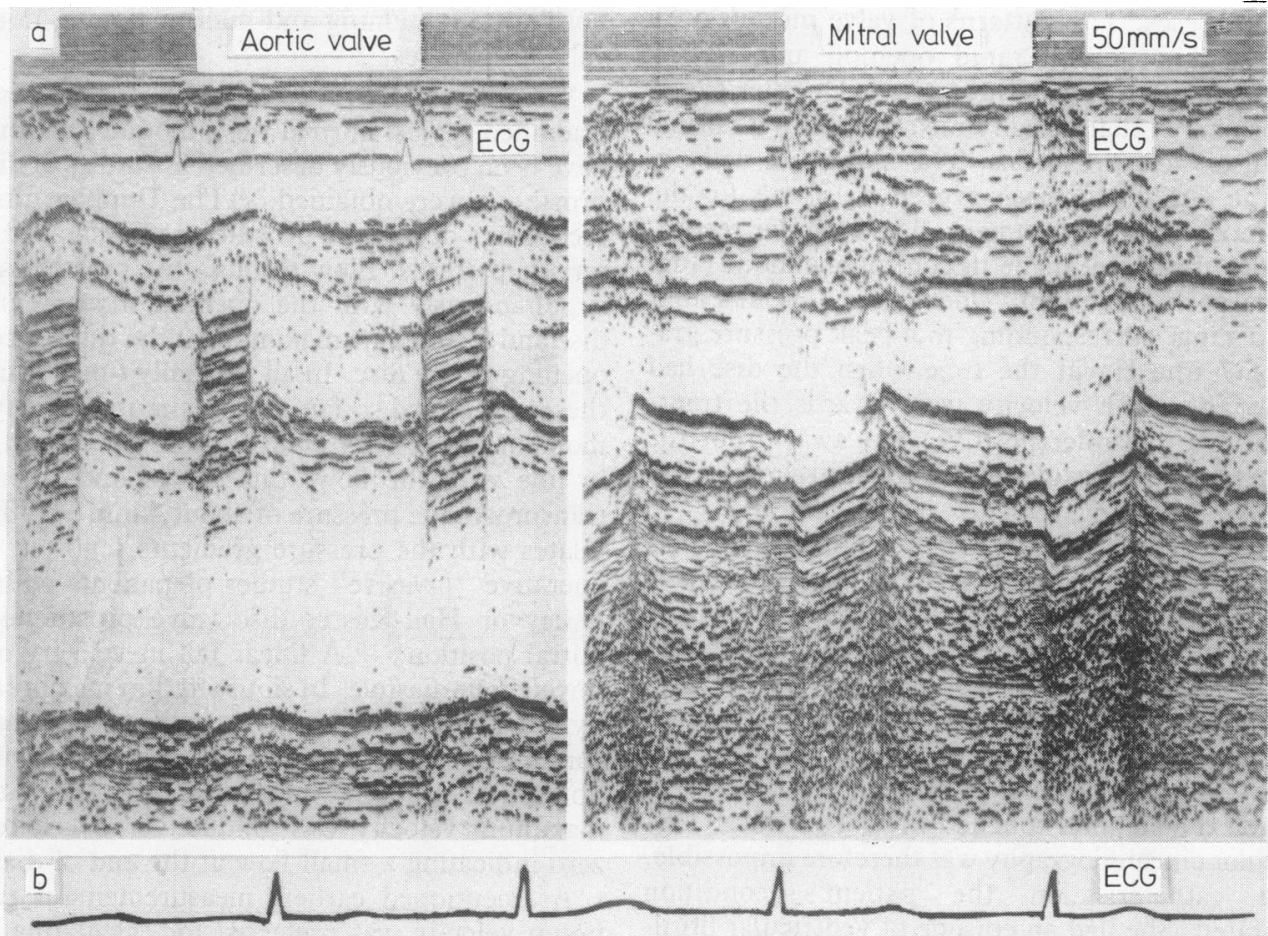

$50 \mathrm{~mm} / \mathrm{s}$

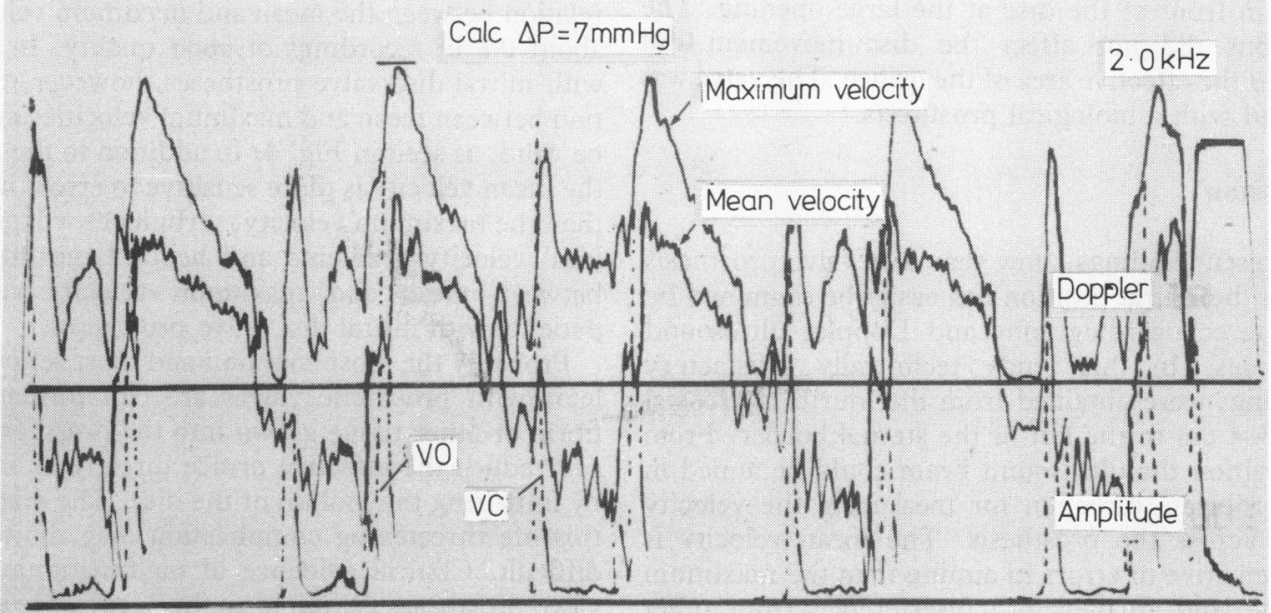

Fig. 7 (a) M mode echocardiogram in a patient (case 5) with a normally functioning Hall-Kaster aortic disc valve prosthesis (No 25) (left panel) and a malfunctioning Hall-Kaster mitral disc valve prosthesis (No 29) (right panel) owing to thrombus formation in the larger orifice of the prosthesis. Note the normal opening and closing of prostheses. (b) Continuous Doppler ultrasound recordings from the same patient taken from the mitral area. The peak gradient across the mitral valve prosthesis was $7 \mathrm{~mm} \mathrm{Hg}$. VO, mitral valve opening; VC, mitral valve closure; ECG, electrocardiogram; Calc $\Delta P$, calculated $\Delta P$ (as in Fig. 3). 
valve prostheses. The patterns of valve motion were normal with a normal rapid opening and closing movement of the discs. The audible Doppler signal was more harsh than usually recorded from a normal mitral disc valve prosthesis. The maximum velocity across the mitral prosthesis exceeded $1.0 \mathrm{~m} / \mathrm{s}$ for the pulsed mode of the instrument. The Doppler recordings were, therefore, made in continuous mode (Fig. $7 \mathrm{~b}$ ). The peak maximum velocity was $1.34 \mathrm{~m} / \mathrm{s}$ after valve opening, corresponding to a peak pressure gradient of $7 \mathrm{~mm} \mathrm{Hg}$ at the time when the disc had opened. The mean velocity was towards the transducer during diastole (positive) but away from the transducer during systole, indicating mitral regurgitation.

Cineangiograms in different projections were performed to study the movement of the disc. The disc in the Hall-Kaster prosthesis is radiopaque. The aortic as well as the mitral disc seemed to move freely with normal opening angles. Injections of contrast material in the aortic root showed no significant regurgitation. Retrograde catheterisation of the left ventricle was different because of serious arrhythmias occurring each time the catheter touched the aortic valve. Left ventricular cineangiography was therefore impossible. During catheterisation the patient's condition deteriorated. She had an episode of ventricular fibrillation, which was cardioverted. She was subsequently comatose and was taken immediately to the operating theatre. At surgery a mitral thrombus formation was found in front of the disc at the large opening. The thrombus did not affect the disc movement but reduced the effective area of the orifice. The valve was replaced with a biological prosthesis.

\section{Discussion}

The present findings show that disc valve prosthesis used in the mitral position can easily be examined by $M$ mode echocardiography and Doppler ultrasound techniques. In this study technically satisfactory recordings were obtained from the fourth intercostal space $3-4 \mathrm{~cm}$ to the left of the sternal border. From this position the ultrasound beam could be aimed in an appropriate direction for measuring the velocity profile across the prosthesis. The mean velocity is more sensitive to errors in aiming than the maximum velocity, and furthermore interference from other vessels may reduce the mean velocity. Maximum velocity is less sensitive to errors in aiming and has therefore been used in preference to calculating changes in flow. Because the prosthetic valve produces strong echoes $M$ mode echograms could be obtained simultaneously from the same position. From the findings so far the combination of the two methods seems to produce data that can be used to distinguish between normally functioning and malfunctioning mitral disc valve prostheses.

When normally functioning valve prostheses were examined typical normal valve motion patterns which have been previously described in studies of disc valve prosthesis were obtained. ${ }^{78}$ The Doppler ultrasound recordings of normal prostheses also showed characteristic patterns. Flow through the prosthesis started simultaneously with the opening movement of the disc and reached its maximum at the time of complete opening of the disc. In all normally functioning prostheses examined so far, the maximum velocity at this moment was about $1.00 \mathrm{~m} / \mathrm{s}$. If the ultrasound beam is in line with the flow, this corresponds to a fall in transprosthetic pressure of about $4 \mathrm{~mm} \mathrm{Hg}$. This correlates with the pressure gradients reported in postoperative "invasive" studies of patients with BjörkShiley or Hall-Kaster disc valve prostheses in the mitral position. $9^{10} \mathrm{~A}$ linear fall in velocity occurred throughout diastole. In sinus rhythm maximum velocity was increased after atrial contraction. Beat to beat variations were usually small. In patients with atrial fibrillation and slow ventricular rate, the initial high maximum velocity was gradually reduced to nearly zero indicating a small flow at the end of diastole.

As mentioned earlier, measurement of the maximum velocity was preferred for calculating changes in flow. If the velocity profile across the cross section of a vessel is completely flat, the mean and maximum velocities will be the same. In our experience, the relation between the mean and maximum velocities is about $\mathbf{0 . 8}$ in recordings of good quality. In patients with mitral disc valve prostheses, however, this relation between mean and maximum velocities might not be valid, as seen in Fig. 4. In addition to the fact that the mean velocity is more sensitive to errors in aiming than the maximum velocity, turbulence will give large local velocity gradients and hence large differences between mean and maximum velocity values in patients with mitral disc valve prostheses.

Probably the most common and most serious problems with prosthetic valves are clot formation and fibrin or other tissue grown into the prosthesis; these can reduce the effective orifice directly or indirectly by impairing the motion of the disc. The diagnosis of this life threatening complication may, however, be difficult. Clinical evidence of malfunctioning mitral valve prostheses is limited to the occurrence of a pansystolic murmur and missing prosthetic clicks. The presence or absence as well as the loudness of the pansystolic murmur is unreliable as the murmur may diminish or disappear with increasing severity of the regurgitation, and opening and closing sounds may be heard despite thrombus formation.

Thrombus formation may be detected by the presence of an echoproducing mass in the vicinity of the 
prosthetic valve without any disc or ball motion. ${ }^{11} 12$ The clot cannot always be clearly identified echocardiographically, however, and multiple echoes from the valve itself or artifacts resulting from the beam transecting both the prosthesis and the tissue beside the valve may be misleading.

Echocardiographic alterations in excursion of the disc have also been reported as one method of detecting malfunction of mitral valve prostheses due to thrombus formation.13-16 A typical finding has a reduced speed of opening and closing of the disc, resulting in a typical "rounded" appearance of the echocardiographic recordings of the prosthesis in diastole. Because of individual variation in the echocardiographic appearance of the normally functioning prosthesis and the importance of critical angulation and positioning of the transducer, it may be difficult to use any of the measurements mentioned above.

As is evident in this study the site of the thrombus must be taken into consideration when the sensitivity of echocardiography in detecting thrombus formation in patients with disc valve prostheses is assessed. In two of the three patients with valve malfunction in this study, the thrombus was located at the smaller orifice behind the disc. The typical echocardiographic findings of delayed opening and closing movements were evident in these patients. In the third patient the thrombus was located at the large orifice in front of the disc and did not interfere with disc motion which resulted in a normal echocardiographic pattern.

The distinctive features of Doppler ultrasound in detecting abnormal prosthetic function have been described in the three cases reported. With this technique all three cases of valve thrombus formation could be differentiated from those with normally functioning prostheses. Typical findings in the former patients were significantly higher values for peak maximum velocity across the malfunctioning prosthesis in diastole. The high velocities in these patients must have been due to the jet caused by the reduction in the actual area of the valve orifice by the thrombus itself. The highest velocities were recorded in the two patients in whom the thrombus was located at the smaller orifice, thus interfering with the movement of the disc. A more moderate increase in maximum flow velocity was found in the third patient, in whom the thrombus was located at the larger orifice in front of the disc.

Even if the form of the velocity curves can give information on the presence or absence of valve regurgitation, ${ }^{17}$ it is my experience that quantifying the degree of regurgitation through a malfunctioning mitral disc valve prosthesis is difficult and sometimes impossible. In general, quantification of valvular insufficiency is only possible if the cross section of the flow area does not change from systole to diastole. This is true in an initial assessment of the case in the aorta or the pulmonary artery, as the arterial diameter shows small changes during the heart cycle. With insufficient tricuspid or mitral valves the valve area is different in systole and diastole. In my experience this variation in valve area may be of particular importance in cases of regurgitation secondary to a malfunctioning mitral disc valve prosthesis with an abnormal backflow pattern through the prosthesis in systole.

\section{References}

1 Holen J, Nitter-Hauge S. Evaluation of obstructive characteristics of mitral disc valve implants with ultrasound Doppler techniques. Acta Med Scand 1977; 201: 429-34.

2 Holen J, Simonsen S, Froysaker T. An ultrasound Doppler technique for the non-invasive determination of the pressure gradient in the Björk-Shiley mitral valve. Circulation 1979; 59: 436-42.

3 Angelsen BAJ. Transcutaneous measurement of aortic blood velocity by ultrasound. A theoretical and experimental approach. Report 75-78-W. Norway: Division of Engineering Cybernetics, Norwegian Institute of Technology, University of Trondheim. 1975.

4 Angelsen BAJ. Analog estimation of the maximum frequency of Doppler spectra in ultrasonic blood velocity measurements. Report 76-21-W. Norway: Division of Engineering Cybernetics, Norwegian Institute of Technology, University of Trondheim, 1976.

5 Brubakk AO, Angelsen BAJ, Hatle L. Diagnosis of valvular heart disease using transcutaneous Doppler ultrasound. Cardiovasc Res 1977; 11: 461-9.

6 Hatle L, Brubakk A, Tromsdal A, Angelsen B. Non-invasive assessment of pressure drop in mitral stenosis by Doppler ultrasound. Br Heart $\mathcal{F}$ 1978; 40: 131-40.

7 Gibson TC, Starek PJK, Moos S, Craige E. Echocardiographic and phonocardiographic characteristics of the Lillehei-Kaster mitral valve prosthesis. Circulation 1974; 49:434-40.

8 Bourdillon PDV, Sharratt GP. Malfunction of Björk-Shiley valve prosthesis in tricuspid position. Br Heart $\mathcal{F}$ 1976; 38: 1149-53.

9 Nitter-Hauge S, Hall KV, Frøysaker T. Mitral valve replacement: A comparative clinical and hemodynamic study of the new Lillehei-Kaster and Björk-Shiley prostheses. Scand $\mathcal{Y}$ Thorac Cardiovasc Surg 1977; 11: 111-7.

10 Nitter-Hauge S, Froysaker T, Hall KV. Clinical and haemodynamic results following replacement of the mitral valve with the new Lillehei-Kaster pivoting disc valve prosthesis. Scand f Thorac Cardiovasc Surg 1977; 11: 15-24.

11 Oliva PB, Johnson ML, Pomerantz M, Levene A. Dysfunction of the Beall mitral prosthesis and its detection by cinefluoroscopy and echocardiography. Am f Cardiol 1973; 31: 393-6.

12 Copans H, Lakier JB, Kinsley RH, Colsen PR, Fritz VU, Barlow JB. Thrombosed Björk-Shiley mitral prostheses. Circulation 1980; 61: 169-74.

13 Pfeifer J, Goldschlager N, Sweatman T, Gerbode F, Selzer A. Malfunction of mitral ball valve prosthesis due to thrombus. $A m \mathcal{F}$ Cardiol 1972; 29: 95-9.

14 Johnson ML, Holmes JH, Paton BC. Echocardiographic determination of mitral disc valve excursion. Circulation 1973; 47: 1274-80.

15 Kawai N, Segal BL, Linhart JW. Delayed opening of Beall mitral prosthetic valve detected by echocardiography. Chest 1975; 67: $239-41$.

16 Clements SD Jr, Perkins JV. Malfunction of a Björk-Shiley prosthetic heart valve in the mitral position producing an abnormal echocardiographic pattern. $\mathscr{F C U} 1$ 1978; 6: 334-6.

17 Tunstall Pedoe DS, MacPherson PC, Meldrum SJ. Absolute intracardiac blood velocities measured with continuous wave Doppler and a new real-time spectral display. In: Lance CT, ed. Echocardiography. The Hague: Martinus Nijhoff, 1979: 77-80.

Requests for reprints to Dr Sigurd Nitter-Hauge, Medical Department B, Laboratory of Cardiology, Rikshospitalet, Oslo 1, Norway. 